\title{
Comparative study of various therapeutic modalities for Guillain Barré syndrome in Assiut University Children Hospital
}

\author{
Zeinab M. Mohy-Eldeen ${ }^{1 \oplus}$, Ahmad R. Ahmad ${ }^{1,2}{ }^{\circ}$, Hayam H. Mahran ${ }^{1 \oplus}$, \\ Khaled Saad ${ }^{10}$ \\ ${ }^{1}$ Departments of Pediatrics, Assiut University Faculty of Medicine, Assiut, Egypt; ${ }^{2}$ Jouf University College of Medicine, Sakaka, Saudi \\ Arabia.
}

\begin{abstract}
Background. Our study aimed to compare the outcome of different therapeutic modalities for the management of children with Guillain-Barré syndrome (GBS) and to identify the associating risk factors that may affect the course and prognosis of the disease.

Methods. Our retrospective study compared the outcomes of different therapeutic regimens for patients with GBS who were admitted to Assiut University Children Hospital, Assiut, Egypt, from 2014 to 2016.

Results. The study included 50 patients diagnosed with GBS. Upper respiratory tract infection was the most prevalent preceding factor (66\%). Acute inflammatory demyelinating polyneuropathy (AIDP) was the most prevalent type $(80 \%)$. Regarding therapeutic modalities, 45 patients started with IVIG treatment, and five patients started plasmapheresis. Seventeen patients showed no improvement after two weeks of IVIG and received plasmapheresis as a sequential therapy. We found no patients who received plasmapheresis, followed by IVIG. Patients treated with plasmapheresis alone showed a significantly shorter duration of hospitalization and better outcomes in comparison to those treated with IVIG alone or with both modalities.
\end{abstract}

Conclusions. AIDP was the most common variety of GBS in our study. GBS patients who were treated with plasmapheresis had a better outcome with a short duration of hospitalization.

Key words: Guillain-Barré syndrome, intravenous immunoglobulin, plasmapheresis, outcome.

Guillain-Barre syndrome (GBS) is an inflammatory polyneuropathy characterized by acute onset, rapid progressive, ascending symmetric muscular weakness, pain, and paresthesia. ${ }^{1,2}$ The disease can take half a day to over four weeks to reach maximum severity and then stabilizes. Weakness varying from abnormal gait to total paralysis, cranial nerve palsies, respiratory compromise, and autonomic instability can be observed., ${ }^{1,2}$ The incidence of GBS in the pediatric age group is 0.8 patients per 100.000. However, the prevalence depends

Khaled Saad

khaled.ali@med.au.edu.eg

Received 12th December 2019, revised 15th January 2020, 15th February 2020, 11st April 2020,

accepted 14th April 2020. on the geographic region. The etiopathogenesis of GBS has been hypothesized to involve a direct immune-mediated mechanism against the peripheral nerve components, including the myelin sheath and the axon. ${ }^{2}$ GBS is a heterogeneous disease with various subtypes. Recognition of these subtypes is of clinical importance since each subtype differs in pathogenesis and prognosis: these include acute inflammatory demyelinating polyneuropathy (AIDP), acute motor axonal neuropathy (AMAN), and unclassified. ${ }^{3}$ Miller Fisher syndrome (MFS) is a clinical variant of GBS characterized by acute onset ophthalmoplegia, ataxia, and areflexia. ${ }^{4}$

The diagnostic criteria of GBS depend on findings such as rapidly progressive and 
relatively symmetrical weakness, muscle paralysis, reduced reflexes, the absence of concomitant fever and exclusion of another possible cause. ${ }^{4}$ Cerebrospinal fluid analysis (CSF) and nerve conduction velocity (NCV) studies are supportive investigations commonly performed in the diagnosis of GBS. Testing for antiganglioside antibodies is performed when available. Blood tests are generally performed to exclude the possibility of other causes for weakness. ${ }^{4}$ Characteristic CSF findings are an elevated protein level usually $>0.55 \mathrm{~g} / \mathrm{L}$ and fewer than ten white blood cells per cubic millimeter (i.e., albumino-cytological dissociation). This combination distinguishes GBS from other conditions (such as lymphoma and poliomyelitis) in which both the protein and the cell count are elevated. Elevated CSF protein levels are found in approximately 50\% of patients in the first three days after onset of weakness, and up to $80 \%$ after the first week. ${ }^{5}$ Electromyography (EMG) and NCV are performed when needed to exclude other causes of acute muscle weakness and to distinguish the different types of GBS. ${ }^{4}$ Plasmapheresis, and intravenous immunoglobulin (IVIG) are the two main treatments for GBS. Plasmapheresis attempts to reduce the body's attack on the nervous system by filtering antibodies out of the bloodstream. Similarly, the administration of IVIG neutralizes pathogenic antibodies and suppresses inflammation through multifactorial mechanisms. The immunomodulatory effect of IVIG is mediated through increasing glycosylation of the Fc portion of serum immunoglobulins which inhibits complement deposition. ${ }^{5}$ Our study aimed to compare the outcome of different therapeutic modalities for the management of children with GBS and to identify the associating risk factors that may affect the course and prognosis of the disease.

\section{Material and Methods}

The Ethics Committee of Assiut University, Assiut, Egypt, approved the study. All methods and procedures used in this study were approved by the Institutional Review Board (IRB number 12-2014).

This retrospective study included all children with GBS where complete, valid records were available in our center. We studied the outcome of different therapeutic regimens for GBS patients who were admitted to Assiut University Children Hospital, Faculty of Medicine, Assiut, Egypt, from June 2014 to June 2016. The diagnosis of acute GBS was based on Brighton criteria. ${ }^{6} \mathrm{We}$ excluded any patient with incomplete data and referred patients with previous treatment trials. We studied all data of GBS, children including history considering age, gender, the season of affliction, antecedent infection, vaccination, neurological deficit, and naïve/recurrent. The clinical data included full neurological examination and laboratory investigations that included CSF examination, NCV, EMG, complete blood count, serum electrolytes ( $\mathrm{Na}$, $\mathrm{K}, \mathrm{Ca}$, and $\mathrm{Mg}$ ), blood chemistry (urea and creatinine), coagulation profile, monitoring of blood gases for patients who developed clinical features of respiratory distress, monitoring while the patient is on plasmapheresis of vital signs, blood pressure, oxygen saturation, and any complications. The severity of the condition was assessed into three categories; Group I (mild GBS): patients with involvement of upper limbs (UL) and lower limbs (LL) only and throughout $>48$ hours, Group II (moderate GBS): patients with involvement of UL and LL throughout $<48$ hours with no bulbar or autonomic manifestation, or, the involvement of UL and LL throughout $>48$ hours with bulbar or autonomic manifestation, and Group III (severe GBS): patients with respiratory muscle involvement at presentation, or UL and LL over $<48$ hours with autonomic manifestation and/or bulbar involvement. ${ }^{7}$ For the treatment of GBS, IVIG or plasmapheresis were used in our center, IVIG in a dose of $0.4 \mathrm{~g} / \mathrm{kg} /$ day for five days, and plasmapheresis according to the protocol of the North American Trial where total 200-250 ml/ $\mathrm{kg}$ is exchanged over 7-10 days. ${ }^{8}$ Patients who received IVIG with no improvement within two weeks received additional plasmapheresis. 
Indicators of improvement included: duration of hospital stay, improvement of movement, weaning of mechanical ventilation, and associated complications.

\section{Statistical analysis}

We used SPSS (version 21, Inc., Chicago, IL, USA) for analysis of patients' data. Categorical data are presented as proportions and continuous data as means \pm SD (standard deviations). P-value $<0.05$ was considered significant. We used the chi-square test to compare the proportions of categorical variables. Finally, a multi-factor logistic regression analysis was done to recognize the statistically significant risk factors that affect the outcome of GBS patients.

\section{Results}

Demographic and clinical data of the patients are presented in Table I. Spring was the highest season of presentation (46\%). Upper respiratory tract infections were the antecedent infection in the majority of patients $(66 \%)$, followed by gastrointestinal infection (34\%). Regarding clinical presentations (Table I), limb weakness was present in all patients (100\%), bulbar manifestations in $72 \%$, respiratory muscle affection in $44 \%$, and facial palsy and ataxia in $2 \%$ each. According to electrophysiological studies, AIDP was the most prevalent type (80\%). Axonal type (AMAN) was detected in $18 \%$ and MFS in $2 \%$ of patients. Regarding treatment modalities, 45 patients started with IVIG treatment, and five patients started with plasmapheresis. Seventeen/45 patients showed no improvement after two weeks of IVIG and received plasmapheresis as a sequential therapy. No patients received IVIG after plasmapheresis. All patients who received plasmapheresis alone started the treatment within one week of disease. About two-thirds of IVIG group (31/45) started the therapy within one week of the disease, and 14 patients began the IVIG after seven days of the clinical manifestations. In our study, the severity of GBS according to Sejvar et al. ${ }^{6}$ was: mild GBS $(n=14)$, moderate GBS $(n=14)$, and severe GBS $(n=22)$ (Tables I, II).

The documented side effects in IVIG included mild infusion reactions, namely, headache (three patients), myalgia (two patients), paresthesia (one patient), and anaphylaxis with hypotension in (one patient). The most important side effects in plasmapheresis group were hypotension (one patient) and arrhythmias (one patient). In patients who received both treatment modalities; sepsis and disseminated intravascular coagulation (one patient) and prolonged fever (five patients) were observed. Regarding outcome, although all patients treated with plasmapheresis alone had severe GBS, they showed a significantly shorter hospital stay and better recovery.

Patients treated with both IVIG and plasmapheresis showed a higher percentage of complications, need for mechanical ventilation, longer hospital stay and higher incidence of deaths in comparison to the other patients.

Regarding risk factors affecting the outcome, the nature of preceding infections was significantly effective: gastrointestinal infections were associated with a longer hospital stay in comparison to respiratory infections. The severe form of GBS was significantly associated with a higher frequency of need for ICU $(\mathrm{p}<0.001)$, mechanical ventilation $(\mathrm{p}<0.001)$, the incidence of complications $(\mathrm{p}=0.02)$, and deaths (0.029). The axonal type was significantly associated with a higher risk of longer hospital stay, need for mechanical ventilation, and ICU admission and death rate (Table III).

\section{Discussion}

GBS is the most important cause of acute flaccid paralysis in children and infants. The mean age and distribution of gender in our series are similar to others. ${ }^{9}$ In our study; males were affected more than females (56\% vs $44 \%$ ) with male to female ratio of $1.27: 1$. In contrast, other studies reported female predominance or equal distribution. ${ }^{10}$ The higher frequency of GBS in 
Table I. Demographic, electrophysiological criteria and clinical data of all studied patients.

\begin{tabular}{lcc}
\hline Parameters & & $\mathrm{N}(\%)$ \\
\hline Age: $<5$ years/>5 years & & $24(48) / 26(52)$ \\
Gender: male/female & & $28(56) / 22(44)$ \\
Residence: rural/urban & & $33(66) / 17(34)$ \\
First attack/relapse & & $48(96) / 2(4)$ \\
Seasonal variation & Spring & $32(46)$ \\
& Summer & $11(22)$ \\
& Winter & $9(18)$ \\
Antecedent infection & Autumn & $7(14)$ \\
& Upper respiratory tract infection & $33(66)$ \\
Clinical data & Gastroenteritis & $17(34)$ \\
& Flaccid paralysis & $50(100)$ \\
& Bulbar manifestations & $36(72)$ \\
Difficulty in respiration & $22(44)$ \\
Subtypes & Gasping respiration & $16(32)$ \\
& Autonomic manifestations & $10(20)$ \\
Treatment groups & Facial palsy & $1(2)$ \\
& Ataxia & $1(2)$ \\
& AIDP & $40(80)$ \\
& AMAN & $9(18)$ \\
& MFS & $1(2)$ \\
& IVIG & $28(56)$ \\
\hline AIDP: & $5(10)$ \\
& Plasmapheresis & $17(34)$ \\
\hline
\end{tabular}

AIDP: acute inflammatory demyelinating polyneuropathy, AMAN: acute motor axonal neuropathy, IVIG: intravenous immunoglobulin, MFS: Miller Fisher syndrome.

females may be related to the age of patients (prepubertal) and type of GBS (AIDP). ${ }^{10}$

In our study, $66 \%$ of patients were from rural areas that are consistent with other reports. ${ }^{11}$ The higher incidence of GBS among rural children could be explained by a higher rate of gastroenteritis and respiratory infections. Although GBS occurred throughout the year, with peaks reported at different seasons, most of the researchers noted a seasonal variation in their studies. ${ }^{11}$ Seasonal variations may depend on the seasonality of the precipitating infection, for instance by the higher frequency of viral infections in Spring in Egypt.9 This explanation is supported by the fact that $66 \%$ of our patients had a history of preceding viral infections. Dhadke et al. ${ }^{12}$ reported respiratory infections as the most common preceding infections in their study, followed by gastroenteritis. In agreement with our findings, a previous study showed that preceding infections were present among $66.5 \%$ of patients of GBS. ${ }^{13}$

In this study, $4 \%$ of patients had recurrent GBS attacks. Koul et al. ${ }^{14}$ reported a recurrence rate of $9.8 \%$ among their patients. GBS may recur in up to $10 \%$ of patients after several months to several years of the initial attack. The risk factors for recurrent GBS include young age, having MFS and patients with a shorter initial episode. ${ }^{14,15}$

In the present study, AIDP was the most common type, like in other studies from Africa, Europe and North America. ${ }^{9,16,17}$ On the other hand, AMAN pattern was the predominant GBS type in Japan, China, and South America. ${ }^{16,17}$ 
Table II. Relation between the type of therapy and outcome of the studied groups.

\begin{tabular}{|c|c|c|c|c|c|}
\hline \multicolumn{2}{|l|}{ Complications } & IVIG $(\mathrm{N}=28)$ & $\begin{array}{l}\text { Plasmapheresis } \\
\qquad(\mathrm{N}=5)\end{array}$ & $\begin{array}{c}\text { IVIG + Plasmapheresis } \\
\qquad(\mathrm{N}=17)\end{array}$ & p-value \\
\hline \multicolumn{2}{|c|}{ Complications: Yes/No, N (\%) } & $10(35.7) / 18(64.3)$ & $2(40.0) / 3(60.0)$ & $15(88.2) / 2(11.8)$ & 0.002 \\
\hline \multicolumn{2}{|c|}{$\begin{array}{l}\text { Need for mechanical ventilation: } \\
\text { Yes/No, (N/\%) }\end{array}$} & $4(14.3) / 24(85.7)$ & $5(100.0) / 0(0.0)$ & $17(100.0) / 0(0.0)$ & $<0.001$ \\
\hline \multicolumn{2}{|c|}{$\begin{array}{l}\text { Duration of hospital stay: } \\
\leq 14 \text { days/>14 days, } N(\%)\end{array}$} & $16(57.1) / 12(42.9)$ & $5(100.0) / 0(0.0)$ & $0(0.0) / 17(100)$ & 0.002 \\
\hline \multicolumn{2}{|c|}{ Outcome: Improved/Died, N (\%) } & $26(92.9) / 2(7.1)$ & $5(100.0) / 0(0.0)$ & $12(70.6) / 5(29.4)$ & 0.072 \\
\hline \multicolumn{2}{|c|}{ Residual neurological deficit, N (\%) } & $4(14.3)$ & $0(0.0)$ & $3(17.6)$ & 0.05 \\
\hline \multirow[t]{3}{*}{ Severity of GBS } & Mild & $14(50.0)$ & $0(0.0)$ & $0(0.0)$ & $<0.001$ \\
\hline & Moderate & $9(32.1)$ & $0(0.0)$ & $5(29.4)$ & \\
\hline & Severe & $5(17.9)$ & $5(100.0)$ & $12(70.6)$ & \\
\hline \multirow[t]{3}{*}{ Pathological type } & Demyelinating & $22(78.6)$ & $4(80.0)$ & $14(82.4)$ & -- \\
\hline & Axonal & 5 (17.9) & $1(20.0)$ & $3(17.6)$ & -- \\
\hline & Miller Fisher & $1(3.6)$ & -- & -- & -- \\
\hline
\end{tabular}

These variations in the types of GBS may be related to genetic factors with no clear evidence clarifying this difference. ${ }^{9}$ Routine electrodiagnostic studies are uncomfortable and can be technologically difficult in children; they should be avoided when the diagnosis of GBS is clear. In addition, they should be undertaken only by experts in pediatric electrophysiological studies. ${ }^{9,16,17}$

In our study, we classified patients according to type of therapy into three groups: IVIG alone, plasmapheresis alone and IVIG followed by plasmapheresis after no improvement observed in two weeks. No patients received IVIG after plasmapheresis. The group treated with plasmapheresis alone showed a shorter duration of hospital stay, mechanical ventilation $<14$ days, and a better outcome, when compared to other groups. All patients of this group received plasmapheresis early within one week of presentation. Our data are in line with previous reports that showed a better outcome of GBS children who received plasmapheresis when compared to other modalities. ${ }^{9,16}$ In contrast to our results, some studies found that IVIG treatment was either better or equivalent to plasmapheresis. ${ }^{18,19}$
IVIG combined with plasmapheresis was associated with a higher frequency of complications, prolonged hospital stay, need for mechanical ventilation, and mortality. This may be due to the higher severity of GBS in this group $(70.6 \%$, Table II) in addition to the delayed starting of management after one week of the manifestations. Oczko-Walker et al. ${ }^{20}$ showed that treatment with IVIG followed by plasmapheresis was not better than IVIG alone. Their patients who received both treatments had a worse GBS disability scale at discharge and had longer hospitalization. ${ }^{20}$ The researchers reasoned such results to a more severe disease course in patients receiving treatment, and/ or, plasmapheresis washing out IVIG and eliminating its therapeutic effect. ${ }^{20}$ The choice to use IVIG or plasmapheresis should be based on several factors, including the accessibility of treatment, the experience of the team, and the patient's condition and comorbidities.

The present study showed no significant effect of age, gender, or residence on the outcome. However, there was statistically significant effect as regards to severity of GBS on admission (need for ICU admission, $\mathrm{p}<0.001$ ), need for mechanical ventilation $(p<0.001)$, and the higher incidence of complications among 


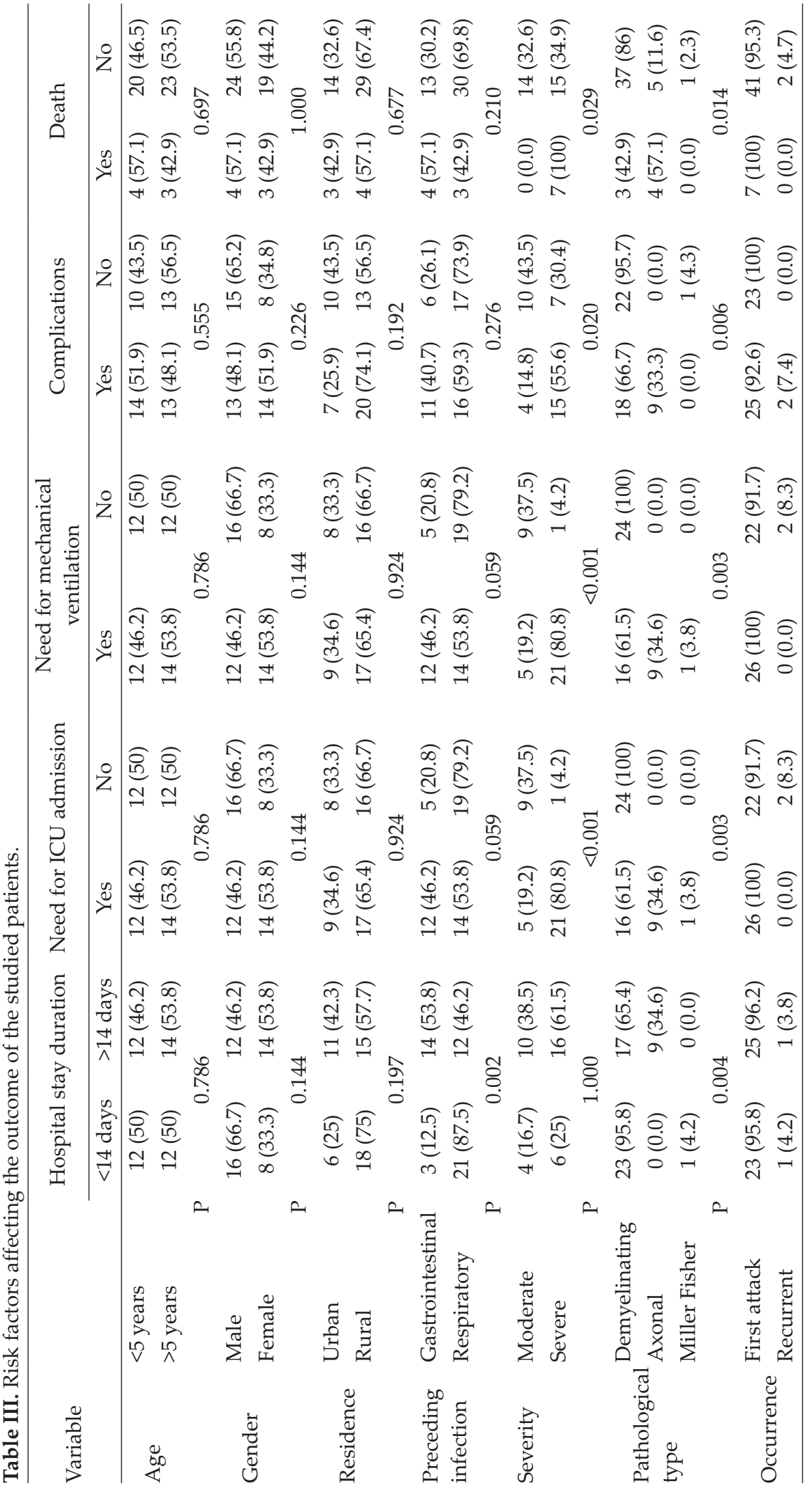


severe patients $(\mathrm{p}=0.02)$, and death $(\mathrm{p}=0.029)$ on outcome of patients with GBS. Our data agreed with previous reports. ${ }^{9,21}$

In our study preceding gastroenteritis, GBS of axonal type were associated with negative outcome indicators such as longer hospital stay, need for ICU admission, need for mechanical ventilation, incidence of complications and mortality. Our results match previous research in Turkey where time to recovery was longer in patients with preceding acute gastroenteritis compared to upper respiratory tract infection. ${ }^{22}$

Our study is retrospective: therefore, some data were not available. Besides, the sample size was small, preventing the application of some comparative statistics. Despite these limitations, it adds epidemiologic and clinical data from this region to a disorder where most information is contributed from East Asia.

Acute inflammatory demyelinating polyneuropathy (AIDP) was the most common variety of GBS in our study. GBS patients who were treated with plasmapheresis had a better outcome with a short duration of hospitalization.

\section{REFERENCES}

1. Rosen BA. Guillain-Barré syndrome. Pediatr Rev 2012; 33: 164-170.

2. Pavone P, Praticò AD, Ruggieri M, et al. Acquired peripheral neuropathy: a report on 20 children. Int J Immunopathol Pharmacol 2012; 25: 513-517.

3. Alexander M, Prabhakar AT, Aaron S, Thomas M, Mathew V, Patil AK. Utility of neurophysiological criteria in Guillain-Barré syndrome: subtype spectrum from a tertiary referral hospital in India. Neurol India 2011; 59: 722-726.

4. Yuki N, Hartung HP. Guillain-Barré syndrome. N Engl J Med 2012; 366: 2294-2304.

5. van den Berg B, Walgaard C, Drenthen J, Fokke C, Jacobs BC, van Doorn PA. Guillain-Barré syndrome: pathogenesis, diagnosis, treatment and prognosis. Nat Rev Neurol 2014; 10: 469-482.
6. Sejvar JJ, Kohl KS, Gidudu J, et al; Brighton Collaboration GBS Working Group. Guillain-Barré syndrome and Fisher syndrome: case definitions and guidelines for collection, analysis, and presentation of immunization safety data. Vaccine 2011; 29: 599612.

7. Raphael JC, Chevret S, Jars-Guincestre MC, Chastang C, Gajdos P. Treatment of Guillain-Barré syndrome by plasma exchange: proposal of a therapeutic strategy. Rev Neurol (Paris) 1996; 152: 359-364.

8. The Guillain-Barré syndrome Study Group. Plasmapheresis and acute Guillain-Barré syndrome. Neurology 1985; 35: 1096-1104.

9. Saad K, Mohamad IL, Abd El-Hamed MA, et al. A comparison between plasmapheresis and intravenous immunoglobulin in children with Guillain-Barré syndrome in Upper Egypt. Ther Adv Neurol Disord 2016; 9: 3-8.

10. Sipilä JOT, Soilu-Hänninen M, Ruuskanen JO, Rautava P, Kytö V. Epidemiology of Guillain-Barré syndrome in Finland 2004-2014. J Peripher Nerv Syst 2017; 22: 440-445.

11. Webb AJS, Brain SAE, Wood R, Rinaldi S, Turner MR. Seasonal variation in Guillain-Barré syndrome: a systematic review, meta-analysis and Oxfordshire cohort study. J Neurol Neurosurg Psychiatry 2015; 8611: 1196-1201.

12. Dhadke SV, Dhadke VN, Bangar SS, Korade MB. Clinical profile of Guillain-Barré syndrome. J Assoc Physicians India 2013; 61: 168-172.

13. Wu X, Shen D, Li T, et al. Distinct clinical characteristics of pediatric Guillain-Barré syndrome: a comparative study between children and adults in Northeast China. PLoS One 2016; 11: e0151611.

14. Koul RL, Alfutaisi A. Prospective study of children with Guillain-Barré syndrome. Indian J Pediatr 2008; 75: 787-790.

15. Mossberg N, Nordin M, Movitz C, et al. The recurrent Guillain-Barré syndrome: a long-term population-based study. Acta Neurol Scand 2012; 126: 154-161.

16. McGrogan A, Madle GC, Seaman HE, de Vries CS. The epidemiology of Guillain-Barré syndrome worldwide. A systematic literature review. Neuroepidemiology 2009; 32: 150-163.

17. Tang J, Dai Y, Li M, et al. Guillain-Barré syndrome in Chinese children: a retrospective analysis. Pediatr Neurol 2011; 45: 233-237.

18. Kuwabara S, Mori M, Ogawara K, et al. Intravenous immunoglobulin therapy for Guillain- Barré syndrome with IgG anti-GM1 antibody. Muscle Nerve 2001; 24: 54-58. 
19. Diener HC, Haupt WF, Kloss TM, et al. A preliminary, randomized, multicenter study comparing intravenous immunoglobulin, plasma exchange, and immune adsorption in Guillain-Barré syndrome. Eur Neurol 2001; 46: 107-109.

20. Oczko-Walker M, Manousakis G, Wang S, Malter JS, Waclawik AJ. Plasma exchange after initial intravenous immunoglobulin treatment in GuillainBarré syndrome: critical reassessment of effectiveness and cost-efficiency. J Clin Neuromuscul Dis 2010; 12: 55-61.
21. $\mathrm{Hu} \mathrm{MH}$, Chen $\mathrm{CM}$, Lin $\mathrm{KL}$, et al. Risk factors of respiratory failure in children with Guillain-Barré syndrome. Pediatr Neonatol 2012; 53: 295-299.

22. Varkal MA, Uzunhan TA, Aydınlı N, Ekici B, Çalışkan M, Özmen M. Pediatric Guillain-Barré syndrome: indicators for a severe course. Ann Indian Acad Neurol 2015; 18: 24-28. 